\title{
Experimental and Theoretical Analysis of an Optical Current Sensor for High Power Systems
}

\author{
A. C. S. BRIGIDA ${ }^{1,2}$, I. M. NASCIMENTO ${ }^{1,3^{*}}$, S. MENDONÇA ${ }^{1,3}$, J. C. W. A. COSTA ${ }^{2}$, \\ M. A. G. MARTINEZ ${ }^{4}$, J. M. BAPTISTA ${ }^{1,5}$, and P. A. S. JORGE ${ }^{1}$ \\ ${ }^{1}$ INESC-TEC (coordinated by INESC Porto), Rua do Campo Alegre, 687. 4169007 Porto, Portugal \\ ${ }^{2}$ Universidade Federal do Pará, Belém, Av. Augusto Correa, 01 - Guamá - 66075-110, Belém, PA, Brazil \\ ${ }^{3}$ Universidade do Porto, Faculdade de Ciencias, Departamento de Física e Astronomia, Rua do Campo Alegre 687, \\ 4169-007 Porto, Portugal \\ ${ }^{4}$ Centro Federal de Educação Tecnológica Celso Suckow da Fonseca, Rio de Janeiro, Brazil \\ ${ }^{5}$ Centro de Competências de Ciências Exactas e de Engenharia, Universidade da Madeira, Funchal, Portugal \\ *Corresponding author: I. M. NASCIMENTO_E-mail: imn@inescporto.pt
}

\begin{abstract}
A magneto-optical sensor, using a dual quadrature polarimetric processing scheme, was evaluated for current metering and protection applications in high voltage lines. Sensor calibration and resolution were obtained in different operational conditions using illumination in the $1550-\mathrm{nm}$ band. Results obtained indicated the feasibility of interrogating such sensor via the optical ground wire (OPGW) link installed in standard high power grids. The polarimetric bulk optical current sensor also was theoretically studied, and the effects of different sources of error considering practical deployment were evaluated. In particular, the interference from external magnetic fields in a tree-phase system was analyzed.
\end{abstract}

Keywords: Fiber optic, optical current sensor, linear birefringence, Faraday effect, tree-phase system

Citation: A. C. S. BRIGIDA, I. M. NASCIMENTO, S. MENDONÇA, J. C. W. A. COSTA, M. A. G. MARTINEZ, J. M. BAPTISTA, and P. A. S. JORGE, "Experimental and Theoretical Analysis of an Optical Current Sensor for High Power Systems," Photonic Sensors, vol. 3, no. 1, pp. 26-34, 2013.

\section{Introduction}

The accurate measurement of electric energy is a fundamental aspect in the development of sustainable energy management systems. In this context, electric current sensors are vital tools nowadays as they allow accurate measurement of consumption and fast identification of failures on power systems [1]. In a high voltage environment, current transformers can be easily damaged by heat, short-circuits or atmospheric electrical discharges, and it is essential to employ protection circuits and insulation, which requires constant maintenance and surveillance. However, standard current transformers used presently in the electric power industry for metering and protection applications have many associated limitations [2]. For instance, due to their high dimension and weight, some of these devices cannot be suspended on the electric line and imply high installation cost $[3,4]$.

The measurement of the electric current through optical methods is appealing for applications in high power systems because it has several advantages over traditional methods, such as the high bandwidth,

Received: 2 August 2012 / Revised version: 15 August 2012

C The Author(s) 2012. This article is published with open access at Springerlink.com 
high electrical insulation, immunity to electromagnetic interference, multiplexing ability, light weight and compatibility with the technology of optical fiber communication.

Most reported optical current sensors have been based on the Faraday effect, or magneto-optical effect [5-7] which is an induced circular birefringence. For a generic polarization state, which can be described as a combination of two orthogonal circular modes (left and right), the application of a magnetic field is translated into the accumulation of a relative phase between the two modes, proportional to the magnetic field. For linearly polarized light, this relative phase results in a rotation of the polarization plane. The Faraday rotation angle $\left(\theta_{t}\right)$, resulting from propagation under the influence of a magnetic field $B$ along a path $L$, is given by

$$
\theta_{t}=\int_{L} V B d L
$$

where $V$ is the Verdet constant $(\mathrm{rad} / \mathrm{m} \cdot \mathrm{T})$, an intrinsic property of the material which depends inversely on the wavelength of the radiation and the temperature $[8,9]$. The Verdet constant is related to the medium properties as described in (2), where $\gamma$ is a constant of the medium called the magneto gyration coefficient, $\lambda$ is the light wavelength, and $n$ is the refractive index of the material [10]:

$$
V \approx \frac{\pi \gamma}{\lambda n} \text {. }
$$

The most used scheme to transform the Faraday rotation in an optical power modulation, easily measurable with a photodetector, is through a polarimetric arrangement. In the simplest configuration, a polarizer is placed at the sensor input in order to set the initial state of polarization of light, and a second polarizer, or analyzer, is placed at the output. The maximum sensitivity is achieved when the angle between the two polarizers is $45^{\circ}$.

\section{Polarimetric sensing scheme}

In this work, a current sensor with a dual quadrature polarimetric detection scheme was implemented and tested, aiming its application at high power systems. The scheme of the configuration tested is represented in Fig. 1.

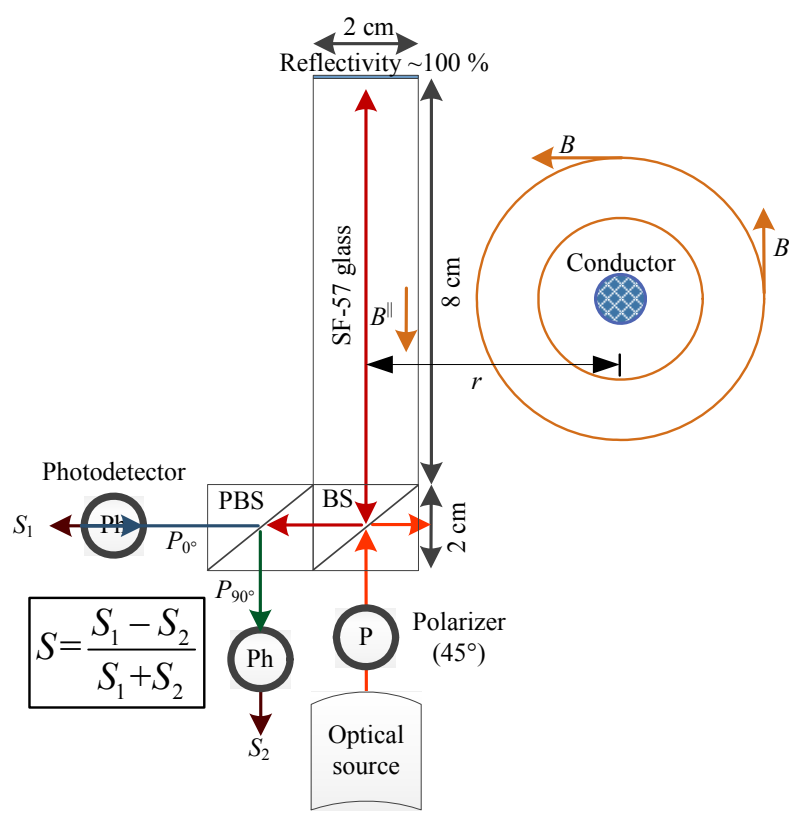

Fig. 1 Bulk optical current sensor with the polarimetric detection scheme.

Linearly polarized light propagates through the sensor element, a Schott glass prism (SF-57), after passing by a beam splitter (BS). While propagating through the prism, the plane of polarization of the light rotates in the presence of a magnetic field. A mirror at the end of the prism reverses the direction of propagation, and the Faraday rotation accumulates due to its non-reciprocal nature. At the output end of the prism, a beam splitter reflects the radiation toward a polarizing beam splitter that separates two orthogonal polarizations in two distinct outputs, $S_{1}$ and $S_{2}$. To obtain maximum sensitivity, the relative orientation between the polarizer and the input polarization has been adjusted to $45^{\circ}$.

The SF-57 glass, in comparison to silica has the particularities of having a high Verdet constant, about 5.5 times greater than that of silica $\left(\mathrm{SiO}_{2}\right)$, approximately $20.07 \mathrm{rad} /(\mathrm{m} \cdot \mathrm{T})$ at $633 \mathrm{~nm}$, very low linear birefringence and a nearly zero elasto-optic 
coefficient.

In this particular experiment, optical sources in the telecom band around $1550 \mathrm{~nm}$ were used instead. This will enable remote measurement at long distances using installed optical ground wire (OPGW) cables. For this spectral region, no Verdet constant value was found in the literature. However, experimental tests conducted with different sources allowed us to estimate a value of about $0.75 \mathrm{rad} /(\mathrm{m} \cdot \mathrm{T})$. This is significantly less than expected considering (2), which, however, is no longer valid in this spectral range.

In a real situation in a high-voltage network, the magnetic field is generated by a conductor perpendicular to the sensor, and the Faraday rotation is given by

$$
\theta=\frac{V \mu_{0} I}{\pi} \arctan \left[\frac{L}{2 r}\right]
$$

where $r$ is the distance between the conductor and the path of propagation of light, $\mu_{0}$ is the magnetic permeability, and $I$ is the current passing through the conductor.

In order to calculate the sensor transfer function, we have performed a Jones matrix analysis. For the sensor shown in Fig. 2, the resulting electric field can be replaced by

$$
\begin{aligned}
& \mathbf{E}_{\text {out } 1}=P\left[45^{\circ}\right] \cdot B S \cdot M \cdot F\left[-\theta_{t}\right] \cdot M \cdot F[\theta] \cdot B S \cdot \mathbf{E}_{\text {in }} \\
& \mathbf{E}_{\text {out } 2}=P\left[45^{\circ}\right] \cdot M \cdot B S \cdot M \cdot F\left[-\theta_{t}\right] \cdot M \cdot F[\theta] \cdot B S \cdot \mathbf{E}_{\text {in }}
\end{aligned}
$$

where $B S, F\left[\theta_{t}\right], F\left[-\theta_{t}\right], M$ and $P[45]$ are the Jones matrices corresponding to the different optical elements used in the setup [11]. In particular, $F\left[\theta_{t}\right]$ accounts for the Faraday rotation effect. In the absence of linear birefringence, this matrix is simply expressed as a coordinate rotation matrix. The change in $\theta_{t}$ signal, after reflection, is necessary in order to account for the non-reciprocity of the Faraday rotation [12]. The polarization rotation is acumulated after reflection rather than canceling out (as in the case of optical activity).

Considering that the intensity reaching each photodetector is given by $I=\mathbf{E}_{\text {out }} \mathbf{E}_{\text {out }}^{*}$, one can easily obtain the sensor transfer function from (4).
The signals in each of the outputs $\left(S_{1}\right.$ and $\left.S_{2}\right)$ are effectively two signals in phase opposition, adequate for the implementation of a sum/difference processing scheme defined by

$$
S=\frac{S_{1}-S_{2}}{S_{1}+S_{2}}=\sin (4 \theta) .
$$

This operation allows eliminating many sources of noise common to the two output signals. In particular, power fluctuations due to the instability of the optical source, disturbances in the fiber connection, vibrations and so on will be canceled out, while still yielding the information about the polarization rotation induced by the magnetic field.

Replacing (3) in (5), using $V=0.75 \mathrm{rad} /(\mathrm{m} \cdot \mathrm{T}), L=$ $8 \mathrm{~cm}$ and $\mu_{0}=4 \pi \times 10^{-7} \mathrm{~N} \cdot \mathrm{A}^{-2}$, the sensor response is obtained as a function of the current and showed in Fig. 2.

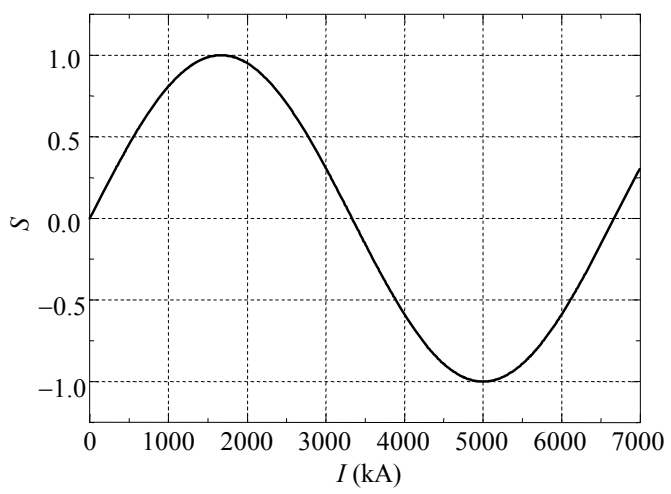

Fig. 2 Sensor estimated transfer function.

It can be seen that the sensor response is linear up to $500 \mathrm{kA}$. Such long range is a consequence of a much lower sensitivity in the infrared spectral range. If the sensitivity is to be enhanced, the lower wavelength must be used instead.

One of the most attractive features of optical current sensors is their high intrinsic bandwidth, which is determined by the radiation time of propagation inside the sensor. The intrinsic bandwidth of this particular sensor can be estimated as the inverse of ten times the propagation time of the light as it passes through the sensor

$$
B=\frac{1}{10 \tau}=\frac{c}{10.2 L n}
$$


with $c=3 \times 10^{8} \mathrm{~m} / \mathrm{s}, L=8 \mathrm{~cm}$ and $n=1.801$ (which is the refractive index of the material at $1570 \mathrm{~nm}$ ), and a sensor intrinsic bandwidth of $104 \mathrm{MHz}$ can be estimated showing potential for measuring very fast phenomena involving high currents, such as the occurrence of transients.

In practice, the sensor system bandwidth will be determined by the lowest bandwidth value of the system elements such as the photodetector or signal detection system, a lock-in or a data acquisition (DAQ) device. When the interest is the measurement application or initial characterization of the sensor response as a function of a well-known current, a lock-in amplifier, with very narrow filtering around the frequency of interest, can be used. If, on the other hand, the idea is to measure very fast current impulses, normally caused by short-circuits in the grid, it will be necessary to use a high speed electronics detection system.

\section{Theoretical analysis}

\subsection{Influence of linear birefringence}

Using the Jones matrix formalism and considering the matrix that describes a sensing material having both linear and circular birefringence[14], it is possible to assess the effect of linear birefringence in the processed signal. In such case, the sensor output is given by

$$
S(\theta, \beta)=2 \theta \frac{\sin \left[2 \sqrt{L^{2} \beta^{2}+4 \theta^{2}}\right]}{\sqrt{L^{2} \beta^{2}+4 \theta^{2}}}
$$

where $\beta$ is the linear birefringence, expressed in radians per meter. From this equation, it can be inferred that the presence of linear birefringence in the sensing material causes a decrease in the sensor sensitivity. In addition, because $\beta$ is dependent on temperature, it can introduce measurement errors. However, when the linear birefringence is much smaller than the Faraday rotation, (7) is simplified to (5). Considering the material used in this experiment had very low intrinsic linear birefringence and also nearly zero elasto-optic coefficient, it is expected that the influence of $\beta$ will be negligible.

In the literature [12], residual birefringence for SF-57 was measured at $633 \mathrm{~nm}$ at $20^{\circ} \mathrm{C}$, for a prism with a length of $10 \mathrm{~cm}$. $\beta$ was found to be approximately $0.384 \mathrm{rad} / \mathrm{m}$ with a temperature dependence of $\delta \beta / \delta T=8.7266 \times 10^{-6} \mathrm{rad} / \mathrm{K}$. Considering the residual birefringence and temperature variations around $50{ }^{\circ} \mathrm{C}$, the total birefringence would be $0.0388 \mathrm{rad} / \mathrm{m}$. The relative errors introduced by such variations in the linear birefringence are calculated by

$$
\operatorname{Error}(\beta)=\left(\frac{\operatorname{Abs}[S(\beta)-S(0)]}{S(0)}\right) \times 100 \% .
$$

In these conditions and considering the intrinsic birefringence axis is aligned with the input polarization (something that could be attained in practice), an error of $0.06 \%$ can be estimated. In practice, the induced birefringence could have a random orientation prone to increase the error. However, a more realistic approach to induced birefringence would be to consider the sensing prism as a succession of smaller prisms with a random size and random birefringence axis orientation that could cancel out partially its deleterious effect. Overall, it is expected that the error will be very small, mainly due to the intrinsic properties of SF-57 that has very low intrinsic linear birefringence and also a nearly zero elasto-optic coefficient (meaning that stress induced birrefringence, due to packaging, will also be very small). It is therefore a very suitable material for implementation of magneto-optic current sensors.

\subsection{Interference of external fields in a tree-phase system}

In a real situation, sensor placement relative to the conductor under measurement is not always perfect. The effect of a linear offset from the maximum sensitivity position can be easily computed however. Considering the sensor is in the vicinity of a conductor carrying a current $I$, arranged as depicted in Fig. 1, but with an offset, $d$, between 
the center of the prism and the conductor, the resulting Faraday rotation is given by $\theta_{i}(r, d, I)=$ $\left(\operatorname{ArcTan}\left[\frac{d+L / 2}{r}\right]-\operatorname{ArcTan}\left[\frac{d-L / 2}{r}\right]\right) V \frac{\mu_{0}}{\pi} \cdot I$.

For $d=0$, as in the case of Fig. 2, (9) is simplified to $(3)$.

In addition, an important problem that needs to be taken into account when using point sensors based on the Faraday effect is the susceptibility of the sensor to perturbations induced by external magnetic fields. In a real application such as in a high voltage grid, the most common situation is the use of a tree-phase system, with a conductor arrangement such as the one shown in Fig. 3. In such case, the currents in each conductor can be described by

$$
\begin{aligned}
& I_{1}=A_{1} \sin \left(2 \pi f_{t}\right) \\
& I_{2}=A_{2} \sin \left(2 \pi f_{t}-\frac{2}{3} \pi\right) \\
& I_{3}=A_{3} \sin \left(2 \pi f_{t}-\frac{4}{3} \pi\right)
\end{aligned}
$$

with $f_{t}$ being the linear frequency of the signal and $A_{n}$ being the amplitude of the current on conductor $n$.

In this configuration, it was intended to analyze the error in measuring the current from conductor 1, based on the local magnetic field, when interfering fields were present, originated by the two other conductors. The operational distances have been defined considering a specific practical situation with $r=0.04 \mathrm{~m}, d_{12}=2.7 \mathrm{~m}$ and $d_{23}=2.73 \mathrm{~m}$.

Also, distinct positions were considered for placement of the sensor. Two different positions in the line were considered [see positions 1 and 2 in Fig. 3(a)]. For the sensor in position 1, it was considered $d_{13}=0 \mathrm{~m}$ and in position $2, d_{13}=d_{12} / 2 \mathrm{~m}$. In addition, for each of these cases, two different geometrical arrangements of the sensor relative to the conductor were considered. The sensor was placed either perpendicular [orientation $Y$ represented in Fig. 3(b)] or parallel [orientation $X$ represented in Fig. 3(c)] to the plane defined by conductors 1 and 2 .

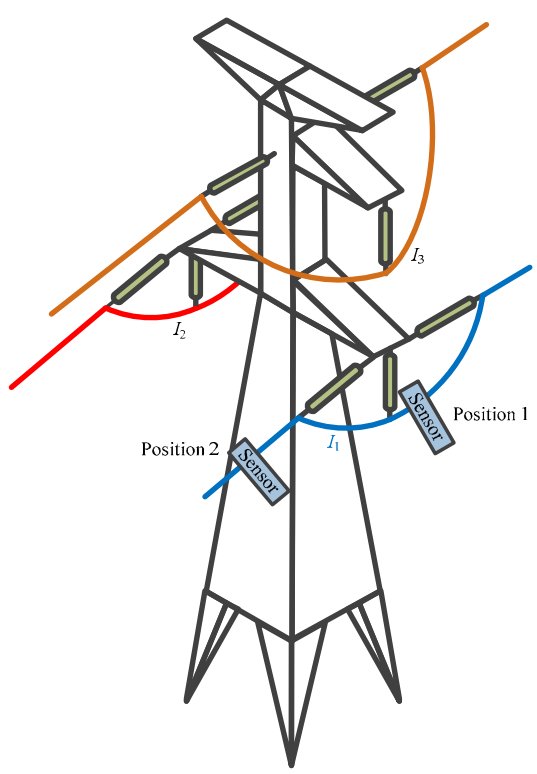

(a)

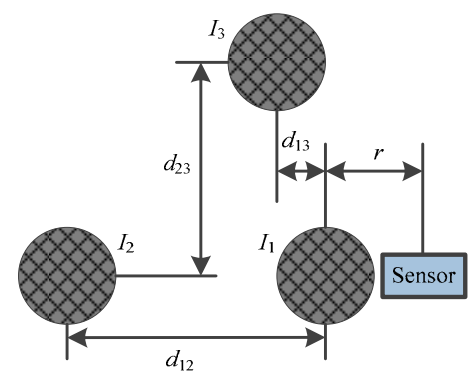

(b)

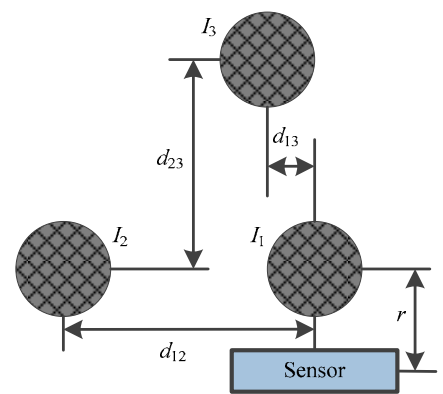

(c)

Fig. 3 Interference of external fields: (a) tree-phase system grid indicating sensor positions 1 and 2, (b) sensors in orientation $Y$, and (c) orientation $X$, to measure the current from the conductor 1 .

For the sensor in orientation $Y$, the total Faraday 
rotation will be given by (11)

$$
\begin{aligned}
\theta & =\theta_{1}\left[r, 0, I_{1}\right]+\theta_{2}\left[\left(r+d_{12}\right), 0, I_{2}\right] \\
& +\theta_{3}\left[\left(r+d_{13}\right), d_{23}, I_{3}\right],
\end{aligned}
$$

and for the sensor in orientation $X$ the total Faraday rotation is instead given by (12)

$$
\theta=\theta_{1}\left[r, 0, I_{1}\right]+\theta_{2}\left[r, d_{12}, I_{2}\right]+\theta_{3}\left[\left(r+d_{23}\right), d_{13}, I_{3}\right]
$$

For the case presented in Figs. 3 (b) and 3(c), the relative errors induced by the interference of the two other conductors (2 and 3) are calculated, by computing

$$
\text { Error }=\frac{S_{p}[0]-S_{p}^{\mathrm{INT}}[0]}{S_{p}[0]} \times 100 \%
$$

where $S_{p}^{\mathrm{INT}}[0]$ and $S_{p}[0]$ represent the peak values of the sensor response with and without interference of the external conductors, respectively. In Fig. 4, the sensor response is shown during one period of the $\mathrm{AC}$ - alternate current waveform with and without interference of external conductors. As expected, the measured amplitude and phase of the signal change when the sensor is subjected to interference from other conductors. Indeed, the measured signal is the result of the superposition of three waves with the same frequency but different relative phases and amplitudes.

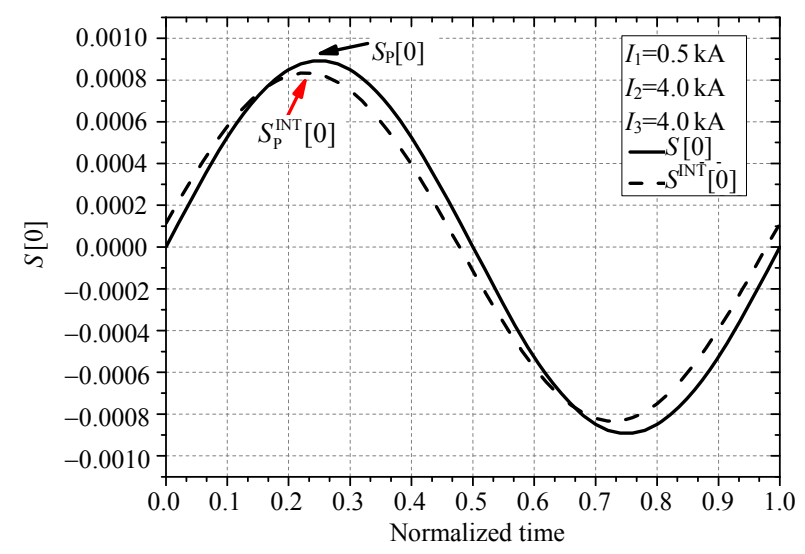

Fig. 4 Snapshot of one period of the sensor response to an $\mathrm{AC}$ current with and without interfering external fields.

In Fig.5(a), it is given a quantitative evaluation of the errors induced in the sensor response considering the first conductor with a current of $0.5 \mathrm{kA}$, and the second and third conductors with currents changing from $0 \mathrm{kA}$ to $4 \mathrm{kA}$. It can be seen that these errors increase rapidly, particularly when the perturbation currents exceed the current to be measured. Nevertheless, it can be seen that for currents of the same magnitude, the most common situation, in a tree-phase system, the induced error is around $1 \%$. The graphs also show important differences for the situations where the sensor is placed in position 1 or 2 with arrangement $Y$ or $X$. It can be seen that the errors are significantly reduced when the sensor is placed parallel to the plane defined by conductors 1 and 2 (orientation $X$ ) particularly when $d_{13}=d_{12} / 2$. In this case, for equal currents in all conductors, the induced error is $0.75 \%$. In a practical application, further reduction in the error introduced by the external field can be attained by using a concentrator of the local field together with insulation for the external fields.

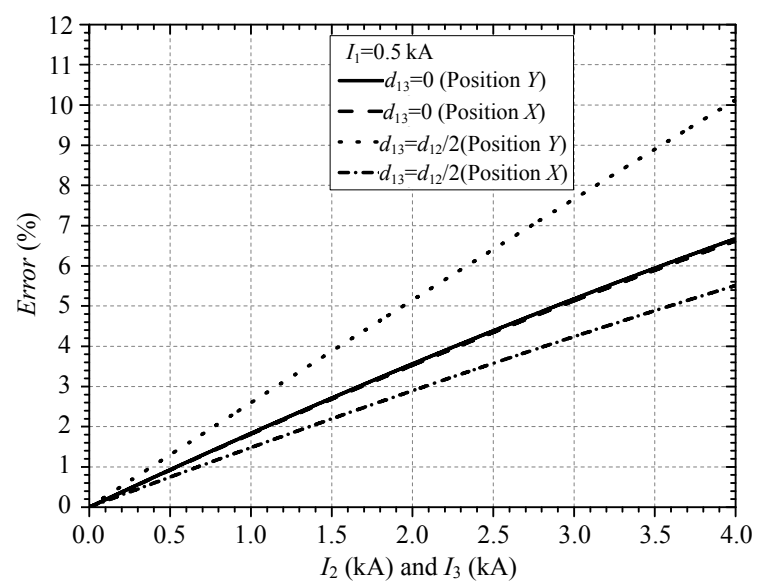

Fig. 5 Errors introduced by the interference of two external conductors for $d_{13}=0$ and $d_{13}=d_{12} / 2$, with $I_{1}=0.5 \mathrm{kA}$.

\section{Experimental results}

The sensor behavior was studied experimentally using a standard broadband erbium doped amplifier (C+L band), with a spectral bandwidth of approximately $100 \mathrm{~nm}$ around $1550 \mathrm{~nm}$. The processed output signal $S$ [see (5)] was obtained using a DAQ NI 6251, with a resolution of $1.92 \mathrm{mV}$, where the signals of each photodetector were compared with a reference signal of the current applied by the magnetic field generator (an inductor). 
The photodetector (Thorlabs PDA 36A) presented a bandwidth of $785 \mathrm{kHz}$ with a gain of $30 \mathrm{~dB}$, and the lock-in (SR850) could be set with integration times, $t_{c}$, from $10 \mu \mathrm{s}$ to $30 \mathrm{ks}$ (bandwidth $\propto 1 / t_{c}$ ).

To simulate large currents occurring in high power systems, the sensor was placed inside an inductor with $N=852$ turns, length $d=47.7 \mathrm{~cm}$, resistance $R=29.599 \Omega$, and inductance $L=21,882$ $\mathrm{mH}$, subjected to a maximum voltage of $7 V_{\text {RMS }}$. The sensor could be submitted to different values of the magnetic field by the control of the current circulating in the inductor. The equivalent current, necessary to obtain the same magnetic field using a single conductor and the setup of Fig. 1, was calculated to be

$$
I_{\text {conductor }} \sim 2072 I_{\text {inductor }} \text {. }
$$

In this way, the behavior of the sensor when placed in the vicinity of a power line conductor could be estimated. To obtain a calibration curve, seven increments of approximately $123 \mathrm{~A}$ of the current at different frequencies $(50 \mathrm{~Hz}, 60 \mathrm{~Hz}, 100$ $\mathrm{Hz}, 250 \mathrm{~Hz}$ and $400 \mathrm{~Hz}$ ) were applied to the sensor. In Fig. 6, the real time response to the step changes in the electric current at $60 \mathrm{~Hz}$ is shown.

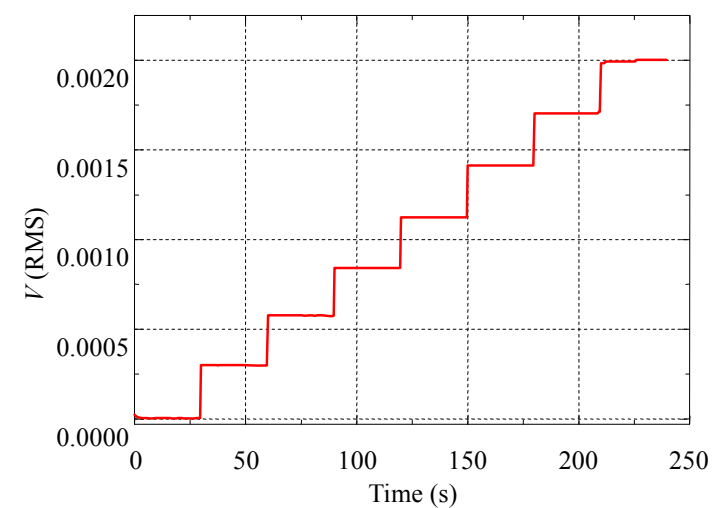

Fig. 6 Response to step changes in the RMS value of an AC electric current at a frequency of $60 \mathrm{~Hz}$.

Each increment was maintained for 30 seconds, during which the sensor output signal was recorded. The value measured in each step was then averaged, and the corresponding values of standard deviation were calculated. With this data, it was then possible to calculate the sensor sensitivity and resolution.
This procedure was repeated at different frequencies and for different current ranges.

In Fig. 7, the sensor response as a function of the applied current at different frequencies can be seen. As expected, the sensor response is linear and fully independent of the operating frequency.

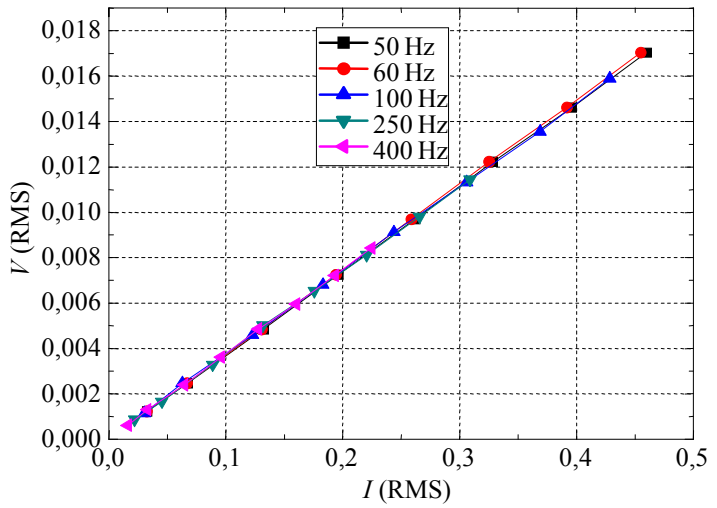

Fig. 7 Sensor response to different frequencies between $50 \mathrm{~Hz}$ and $400 \mathrm{~Hz}$.

Considering the signal acquisition system incorporated with the DAQ and a signal processing program in LabView, the bandwidth defined for the system was set to $125 \mathrm{mHz}$, around the measured signal frequencies of $50 \mathrm{~Hz}, 60 \mathrm{~Hz}$ and $100 \mathrm{~Hz}$. For comparison purposes, measurements were also made using a lock-in amplifier (in this case, a single output signal, $S_{1}$ or $S_{2}$, was measured). The results obtained with the DAQ can be seen in Fig. 8, where the responses of each of the channels $S_{1}$ and $S_{2}$, together with the processed signal, $S$, are shown. The value of the applied current was recalculated, considering (14).

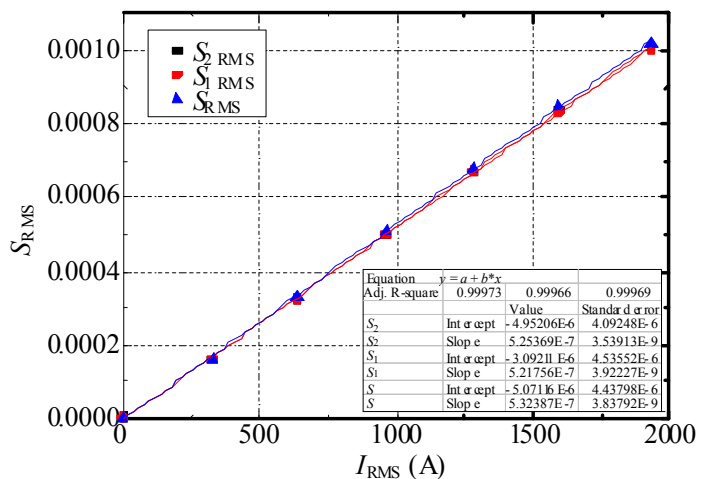

Fig. 8 Response of the sensor outputs as a function of the applied current using an optical source in the 1550-nm band. 
It can be seen that all signals behave in a linear fashion for currents up to $2 \mathrm{kA}$. The additional test were made, where the optical power injected into the sensor was changed during the measurement. In this case, it could be seen that while reducing the optical power down to $36 \%$ of its nominal operation value introduced substantial changes in $S_{1}$ and $S_{2}$, the processed signal $S$ was shown to be independent of such fluctuations.

The sensor resolution was estimated considering that the minimum detectable value was given by two times the standard deviation of the signal. The worst value obtained in the full range of the current measured was considered as the sensor resolution. The resolution was estimated both for the individual signals $S_{1}$ and $S_{2}$ and also for the processed signal $S$ [see (5)]. In Table 1, a comparison is made between the values of the standard resolutions, estimated in the laboratory with the lock-in and with the DAQ for all signals.

Table 1 Optical sensor current resolution.

\begin{tabular}{c|c|c|c|c}
\hline & \multirow{2}{*}{ Lock-in } & \multicolumn{3}{|c}{ DAQ } \\
\cline { 3 - 5 } & & $S_{1}$ & $S_{2}$ & $S$ \\
\hline Resolution (A) & 3.6 & 17.9 & 12.9 & 11.7 \\
\hline Normalized resolution $(\mathrm{A} / \sqrt{ } \mathrm{Hz})$ & 10.2 & 50.6 & 36.6 & 33.1 \\
\hline
\end{tabular}

For the DAQ system, the results demonstrate that the processed signal $S$ has an improved resolution when compared with the results obtained with the signals $S_{1}$ and $S_{2}$. It is expected that in a practical field application the individual signals will deteriorate rapidly due to the environmental perturbations while the processed signal should maintain a more stable behavior. On the other hand, it can be seen that the lock-in acquisition yields an improved resolution, even when considering the non-processed signal. In this particular test to enable the desired signal processing, the signals from the two photodetectors must be read by the DAQ. However, the device used had a resolution in the order of tens of $\mathrm{mV}$ which limited the final resolution attainable by the sensor compared with the results obtained with the lock-in amplifier. Nevertheless, such differences can be overcome with dedicated electronics in order to obtain a better performance.

\section{Conclusions}

In this work, a magneto-optic current sensor with a dual quadrature polarimetric configuration was implemented and tested aiming its application at high voltage environments. The sensor operation was demonstrated, using an optical source in the 1550 -nm band. Such approach is compatible with the standard optical fiber technology, enabling sensor interrogation through the OPGW cables typically used in high power grids. The linear birefringence effect upon an SF-57 bulk optical glass subjected to $50{ }^{\circ} \mathrm{C}$ change in temperature was calculated. A very small error was found due to the intrinsic properties of SF-57. On the other hand, the external magnetic fields in a tree-phase system grid can cause considerable errors depending on the relative currents, distances and positions of the sensors. Nevertheless, it was shown that proper placement of the sensors can be explored to minimize the induced measurement errors.

\section{Acknowledgment}

This work was supported by ANEEL (Agência Nacional de Energia Elétrica) through the research and development program of the companies of the group TBE - Transmissoras Brasileiras de Energia (EATE - Empresa Amazonense de Transmissão de Energia, ECTE - Empresa Catarinense de Transmissão de Energia, ENTE - Empresa Norte de Transmissão de Energia, ERTE - Empresa Regional de Transmissão de Energia, ETEP - Empresa Paraense de Transmissão de Energia, LUMITRANS - Companhia Transmissora de Energia Electrica and STC - Sistemas de Transmissão Catarinense). The authors thank CAPES - Coordination for the Improvement of Higher Education and $\mathrm{CNPq}-$ 
National Council for Scientific and Technological Development for financial support.

Open Access: This article is distributed under the terms of the Creative Commons Attribution License which permits any use, distribution, and reproduction in any medium, provided the original author(s) and source are credited.

\section{References}

[1] H. J. El-Khozondar, M. S. Muller, R. J. El-Khozondar, and A. W. Koch, "Magnetic field inhomogeneity induced on the magneto-optical current sensors," in Information Photonics 2011 ICO International Conference, Ottawa Convention Center, Canada, May 18-20, pp. 1-2, 2011.

[2] F. Rahmatian, "High-voltage current and voltage sensors for a smarter transmission grid and their use in live-line testing and calibration," presented at Power and Energy Society General Meeting IEEE, Minneapolis, Minnesota, USA, July 24-29, 2010.

[3] Z. P. Wang and X. Y. Liu, "Effects of linear birefringence inside sensing head upon bulk glass current sensors sensitivity," Optics \& Laser Technology, vol. 38, no. 3, pp. 177-182, 2006.

[4] G. Li and M. Kong, "Sensitivity improvement of an optical current sensor with enhance Faraday rotation," Journal of Lightwave Technology, vol. 15, no. 12, pp. 2246-2252, 1997.

[5] Y. N. Ning, Z. P. Wang, A. W. Palmer, K. T. V. Grattan, and D. A. Jackson, "Recent progress in optical current sensing techniques," Review Scientific Instrumets, vol. 66, no. 5, pp. 3097-3111, 1995.

[6] A. F. Jenkins and E. H. White, Fundamental of optics. New York: McGraw-Hill Book Company, 1953, pp. 597-598.

[7] F. J. Loeffler, "A Faraday rotation experiment for undergraduate physics laboratory," American Journal of Physics, vol. 51, no. 7, pp. 661-663, 1983.

[8] F. L. Pedrotti and P. Bandettini, "Faraday rotation in the undergraduate advanced laboratory," American Journal of Physics, vol. 58, no. 6, pp. 542-545, 1989.

[9] H. El-Khozondar and M. Muller, "Magnetic field inhomogeneity induced on the magneto-optical current sensors," in Information Photonics 2011 ICO International Conference, Ottawa Convention Center, Canada, May 18-20, pp. 1-2, 2011.

[10] B. E. A. Saleh and M. C. Teich, Fundamentals of Photonics. NewYork: John Wiley \& Sons, Inc., 1991.

[11] N. C Pistoni, "Simplified approach to the Jones calculus in retracing optical circuits," Applied Optics, vol. 34, no. 34, pp. 7870-7876, 1995.

[12] P. A. Williams, A. H. Rose, G. W. Day, T. E. Milner, and M. N. Deeter, "Temperature dependence of the verdet constant in several diamagnetic glasses," Applied Optics, vol. 30, no. 10, pp. 1176-1178, 1991.

[13] G. Li, M. Kong, G. R. Jones, and J. W. Spencer, "Sensitivity improvement of an optical current sensor with enhance Faraday rotation," Journal of Lightwave Technology, vol. 15, no. 12, pp. 2246-2252, 1997.

[14] Z. P. Wang, Q. B. Li, and Q. Wu, "Effects of the temperature features of linear birefringence upon the sensitivity of a bulk glass current sensor," Optics \& Laser Technology, vol. 39, no. 1, pp. 8-12, 2007. 